\title{
¿Innovación en el Perú?: una reflexión a partir de indicadores sintéticos
}

\author{
Jean Pierre Seclén Luna ${ }^{1}$ \\ Departamento Académico de Ciencias de la Gestión \\ Pontifica Universidad Católica del Perú \\ jseclen@pucp.pe \\ Fátima Ponce Regalado \\ Departamento Académico de Ciencias de la Gestión \\ Pontifica Universidad Católica del Perú \\ fponce@pucp.edu.pe
}

Este documento tiene como propósito describir de forma somera y sucinta el estado de la innovación del Perú a partir de indicadores sintéticos que ofrece el Índice Global de Innovación de la Universidad de Cornell, INSEAD, WIPO y el Índice Global de Competitividad del Foro Económico Mundial.

Estos indicadores nos muestran que el Perú se encuentra rezagado en el ranking mundial de innovación, siendo la falta de colaboración para la innovación la principal barrera: la desconfianza y/o aversión al riesgo están presentes en la mayoría de las empresas e instituciones peruanas, de tal forma que nuestro ecosistema de innovación se encuentra desarticulado.

Por otro lado, analizamos la Encuesta Nacional de Innovación de 2012 y 2015 y concluimos que la actividad de innovación en la que invierten más las empresas de manufacturas es la adquisición de bienes de capital. Sin embargo, las actividades de I+D interna y la capacitación del personal son aquellas en las que menos invierten. De esta forma, la dependencia tecnológica es la estrategia más empleada por los empresarios peruanos, quedando en un segundo plano la creación o acumulación de conocimiento interno.

1. Los autores agradecen al Departamento Académico de Ciencias de la Gestión de la PUCP por la ayuda económica brindada para la realización del presente artículo. Asimismo, a Danny Pizarro por el apoyo en el tratamiento de la información de las bases de datos del INEI. 


\section{Introducción}

En las últimas décadas los estudios sobre innovación han adquirido relevancia a nivel internacional, no solo por la importancia de la innovación en la mejora de la competitividad de las organizaciones, sino también por los beneficios que trae a la sociedad en su conjunto. En la actualidad, distintas organizaciones —nacionales e internacionales — realizan reportes anuales sobre el estado de la innovación en los que se puede apreciar la evolución del perfil de los países en cuanto a su grado de competitividad e innovación a partir de diversos marcos teóricos propuestos.

Consideramos que, en nuestro país, se vienen realizando cada vez más investigaciones que intentan llenar el vacío existente al respecto. Este artículo no pretende ser exhaustivo dada la magnitud del asunto; sin embargo, trata de describir de manera somera y sucinta cuál es el estado de la innovación del Perú a partir de indicadores sintéticos que son ofrecidos por el Índice Global de Innovación (GII) de la Universidad de Cornell, INSEAD y WIPO, y el pilar de sofisticación e innovación del Índice Global de Competitividad (GCl) del Foro Económico Mundial.

Asimismo, el análisis se complementa con las actividades de innovación que realizan las empresas de manufacturas reportadas en la Encuesta nacional de innovación en la industria manufacturera (ENIIM) tanto de 2012 como de 2015.

\section{2. Índice de Innovación Global de la Universidad de Cornell, INSEAD y WIPO}

El Índice de Innovación Global (GII, por sus siglas en inglés) es un proyecto que fue lanzado en 2007 por el profesor Soumitra Dutta del INSEAD (The Business School for the World). Este reporte anual es copublicado con el INSEAD y la Organización Mundial de la Propiedad Intelectual (WIPO, por sus siglas en inglés), y cuenta además con un consejo asesor formado por un selecto grupo de líderes y expertos internacionales en innovación. Como se señala en el reporte anual de 2016, "el objetivo de implementar el Gll fue determinar nuevas métricas y aproximaciones que capturen mejor la riqueza de la innovación en la sociedad. [...]. El GIl ayuda a crear un ambiente en el cual los factores de la innovación están bajo continua evaluación, y esto provee una herramienta clave y rica base de datos de métricas detalladas para refinar las políticas de innovación» (p. 49). Ello es importante debido a que la medición de la innovación es compleja; así, Dutta, Lanvin y Wunsch-Vincent señalan que la «innovación no puede ser reducida a inversión en investigación y desarrollo (I+D) y patentes. La visión ofrecida por el Gll es más compleja y ofrece un diferente punto de vista acerca de la dinámica que moldea la innovación a nivel mundial» (Dutta, Lanvin \& Wunsch-Vincent, 2013, p. 3).

El GII, que en 2016 tomó en cuenta a 128 países y 82 indicadores en total, es el promedio simple de dos subíndices: los inputs y outputs de innovación (ver gráfico 1):

a. Input de innovación: formado por cinco pilares que capturan elementos de la economía nacional que permiten desarrollar las actividades de innovación tales como instituciones (entorno político, regulatorio, empresarial), capital humano e investigación (educación, educación terciaria, investigación y desarrollo), infraestructura (nuevas tecnologías de la comunicación, 
infraestructura en general, sostenibilidad ecológica), sofisticación del mercado (créditos, inversiones, comercio, competencia y tamaño del mercado) y sofisticación de los negocios (trabajadores del conocimiento, vínculos para la innovación, absorción de conocimiento).

b. Output de innovación: formado por dos pilares que muestran los resultados de las actividades de innovación en una economía. Outputs de conocimiento y tecnología (creación, impacto y difusión del conocimiento) y outputs de creatividad (activos intangibles, bienes y servicios creativos, creatividad online).

\section{Gráfico 1: Marco conceptual del Índice Global de Innovación 2016}

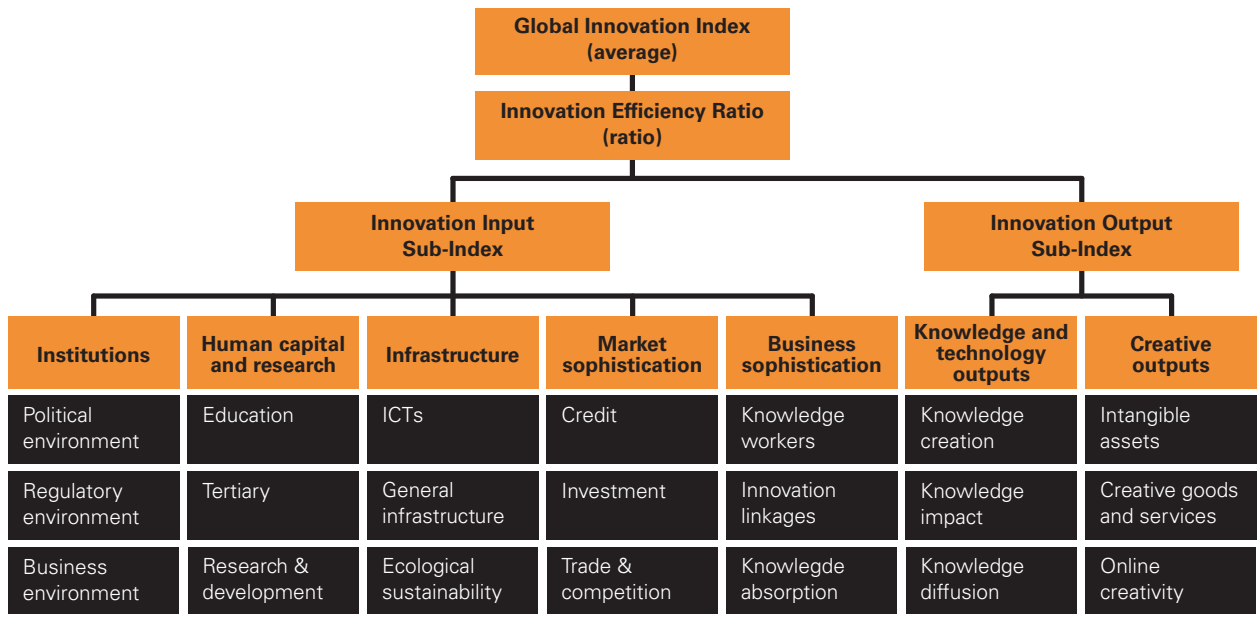

Fuente: Dutta, Lanvin \& Wunsch-Vincent (2016).

En síntesis, el Gll nos permite evaluar cómo es el entorno para llevar a cabo las actividades de innovación. En particular, consideramos importante concentramos en el pilar 5 («Sofisticación de negocio»), ya que trata de capturar el nivel de sofisticación de negocios para asegurar la forma en que las empresas realizan las actividades de innovación. No obstante, para conocer la sofisticación de los negocios, debemos comprender los tres subpilares que la conforman:

- Trabajadores del conocimiento (que incluye indicadores tales como: empleo en servicios intensivos en conocimientos, disponibilidad de formación formal a nivel empresarial, el gasto en I+D realizado por las empresas y el porcentaje de empleo femenino con posgrados).

- Vínculos para la innovación qque incluye la colaboración en investigación universidad-industria, el estado de desarrollo de los clusters, el nivel de I+D financiado por empresas extranjeras, el número de acuerdos joint ventures y las alianzas estratégicas).

- Absorción del conocimiento (relacionado a sectores con alto contenido de tecnología o claves para la innovación, tales como los pagos de licencias y 
regalías como porcentaje del comercio total, importaciones de tecnología de computadoras y servicios TIC como porcentaje del comercio total, flujos netos de las IDE como porcentaje del PBI y el talento en investigación en los negocios).

Ahora bien, tomando en consideración lo explicado, en Perú, durante el periodo 2011-2016, los subpilares de la sofisticación de negocios han presentado una tendencia regular si los analizamos empleando el valor del índice (que va de 0 a 100). Así, a nivel del valor del índice, apreciamos que se presenta un ligero descenso en el índice «Vínculos para la innovación», pero se mantienen casi constantes los indicadores que miden a los trabajadores del conocimiento y la absorción del conocimiento (ver gráfico 2). Estos resultados podrían estar indicando que el entorno para el desarrollo de innovaciones en nuestro país es incipiente, entre otras razones, porque el ecosistema de innovación en el Perú aún sigue desarticulado; y muestra de ello es que las relaciones de colaboración para innovar entre universidad-empresa-Estado siguen siendo débiles (OCDE, 2011; Ísmodes, 2016).

\section{Gráfico 2: Evolución del valor del GII a nivel del pilar «Sofisticación de negocios» (Perú, 2011-2016)}

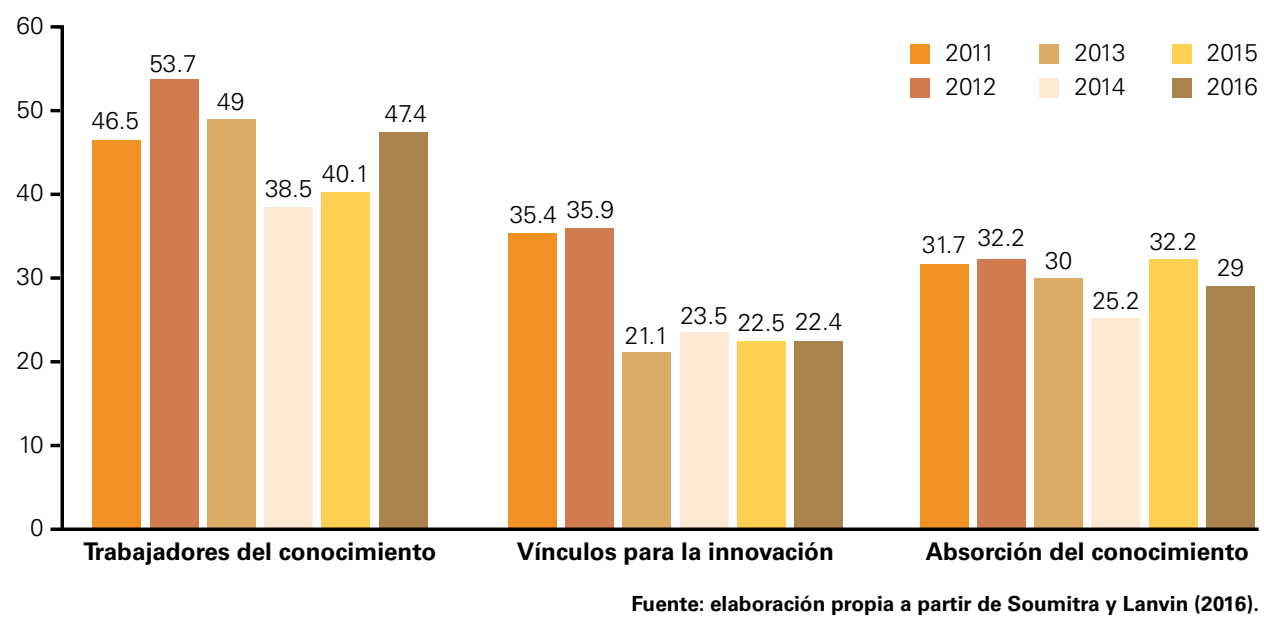

Por otro lado, si analizamos los indicadores de acuerdo a su evolución en el ranking de los indicadores (ver gráfico 3), podemos apreciar que Perú ha perdido posiciones con respecto a otros países, especialmente en el indicador "Vínculos para la innovación», ya que ha pasado del puesto 48 que ocupó en el año 2011 al puesto 97 en 2016. Esto nos reafirma que todos los actores del ecosistema de innovación deben dedicar mayores esfuerzos, ya que a nivel relativo estos siguen siendo escasos. 


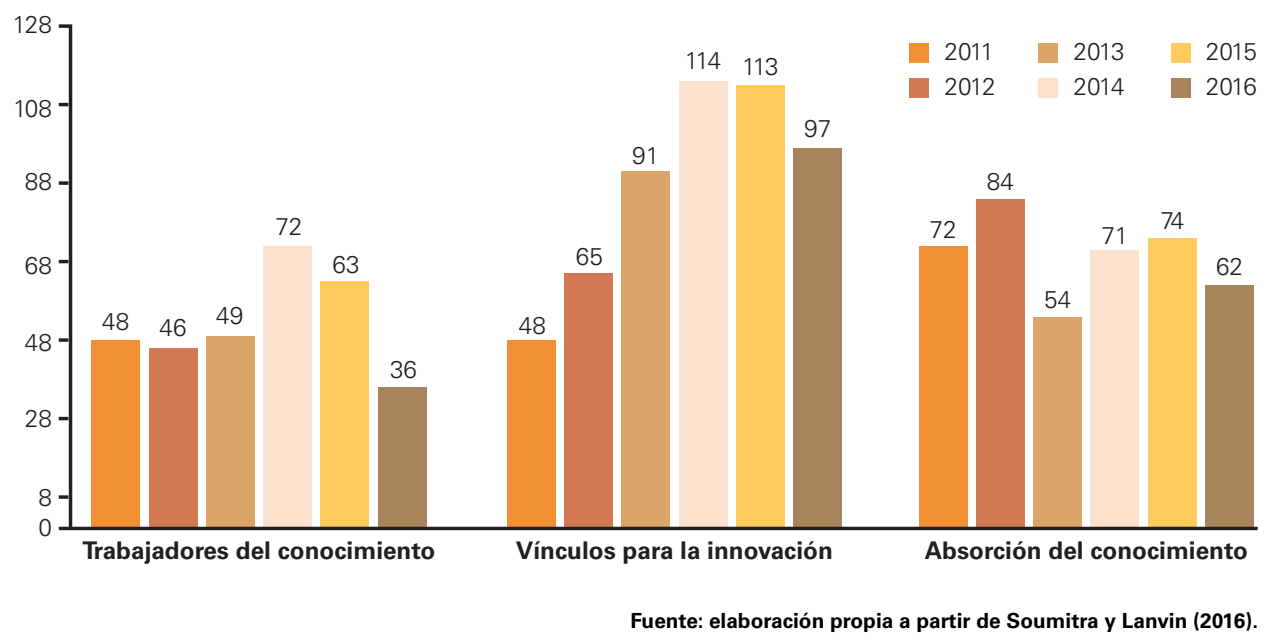

\section{3. Índice Global de Competitividad del Foro Económico Mundial}

Desde 1991, en Ginebra (Suiza), se reúnen anualmente los principales líderes empresariales, políticos, intelectuales y expertos de diversos sectores para analizar los problemas que afronta el mundo. El Foro Económico Mundial (WEF, por sus siglas en inglés) genera una serie de informes de investigación entre los cuales el Índice Global de Competitividad ( $\mathrm{GCl}$, por sus siglas en inglés) adquiere especial relevancia, ya que contiene 12 pilares que miden y reflejan las debilidades y fortalezas que los países poseen en temas relacionados con: 1) requerimientos básicos para el desarrollo, donde se miden las instituciones, infraestructuras, entorno macroeconómico, salud y educación primaria; 2) facilitadores de eficiencia, que mide la educación superior, la eficiencia del mercado de bienes, la eficiencia del mercado laboral, el desarrollo de mercados financieros, la preparación tecnológica y el tamaño del mercado; 3) factores de sofisticación e innovación que miden la sofisticación de los negocios y la innovación (ver gráfico 4). Ahora bien, si ponemos el foco de atención en la innovación, este pilar se mide a partir de siete indicadores: la capacidad de innovación que tienen las empresas, la calidad de las instituciones científicas, el gasto empresarial en I+D, la colaboración universidad-empresa en I+D, las compras públicas de productos de alta tecnología, la disponibilidad de científicos e ingenieros y las aplicaciones de patentes.

De otro lado, los valores de los indicadores se miden en una escala del 1 al 7 , siendo 7 el máximo valor a alcanzar (World Economic Forum, 2016). 


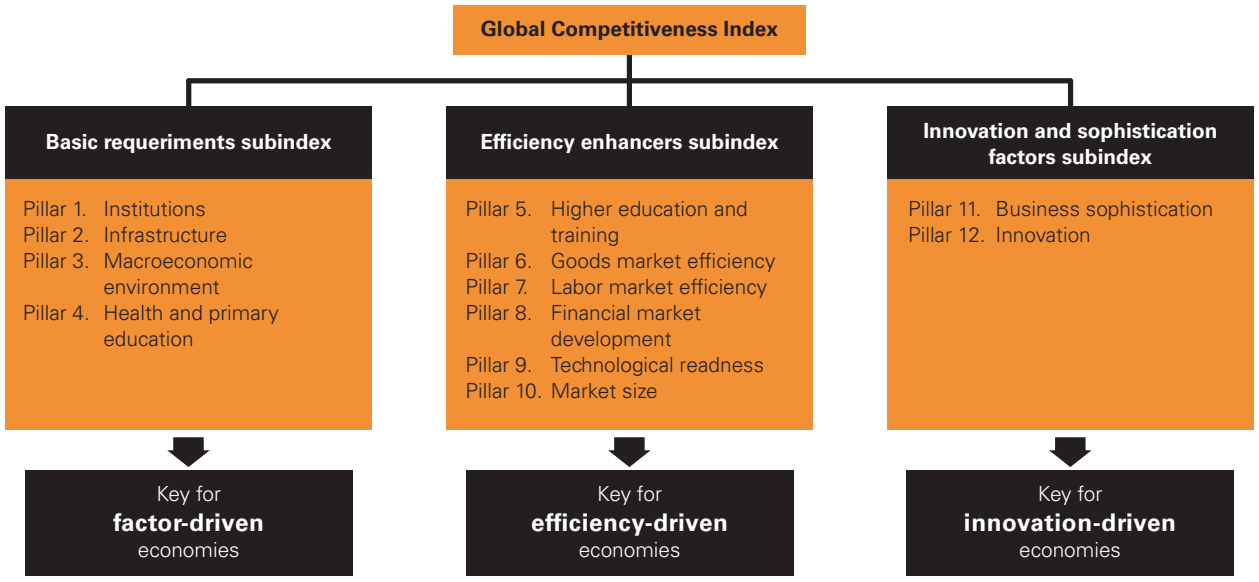

Fuente: World Economic Forum (2016).

La evolución del valor de los indicadores de innovación durante el periodo 20102015 para el caso de Perú presenta un ligero aumento (ver gráfico 5), en particular en la capacidad de innovación, en la calidad de las instituciones de investigación científica y en el gasto empresarial en I+D. No obstante, a pesar de ello, si el análisis lo hacemos tomando en cuenta el ranking de los indicadores, podemos apreciar que Perú ha perdido posiciones con respecto a otros países, lo cual significa que los esfuerzos realizados por los actores de nuestro entorno aún son insuficientes, como ya hemos mencionado. En concreto, y tomando como referencia el año 2010 respecto de 2015, podemos apreciar que la capacidad de innovación del país desciende del puesto 99 al puesto 111. Asimismo, la calidad de las instituciones de investigación científica retrocedió del 109 al puesto 114, el gasto empresarial en I+D de la posición 118 a la 122, la colaboración universidad-empresa en I+D del puesto 103 al 110, las compras públicas de producción de alta tecnología del puesto 98 al 125; y, por último, la disponibilidad de científicos e ingenieros también retrocede de la posición 102 al puesto 114 (ver gráfico 5).

\section{Gráfico 5: Evolución del valor de los indicadores de innovación WEF 2010-2015 (Perú)}

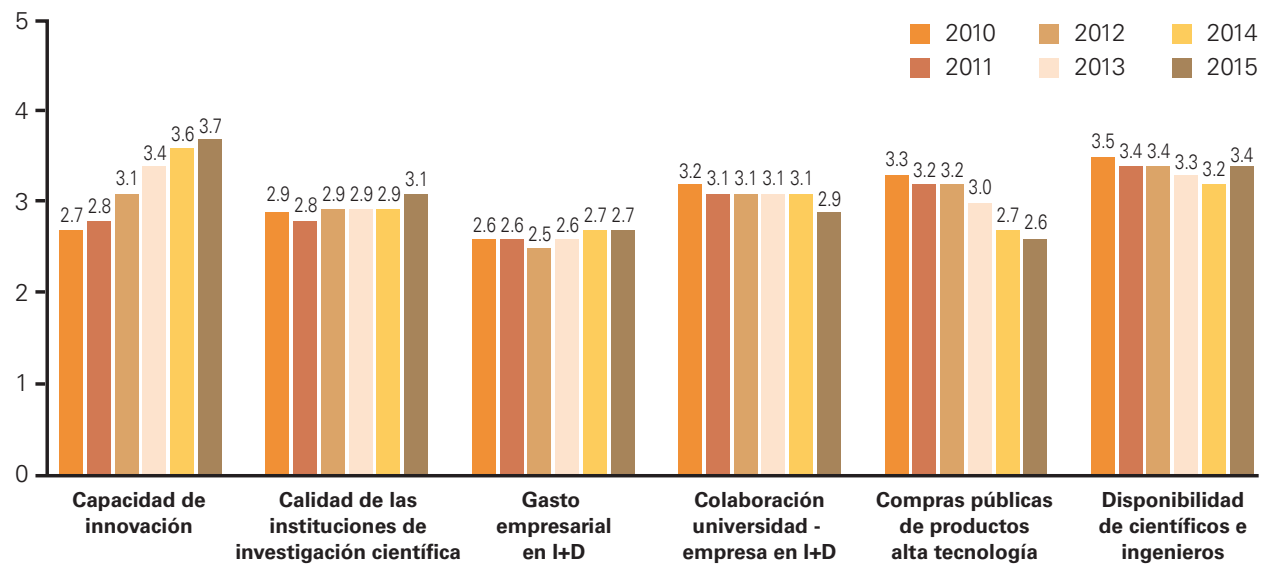

Fuente: elaboración propia a partir del World Economic Forum (2016). 


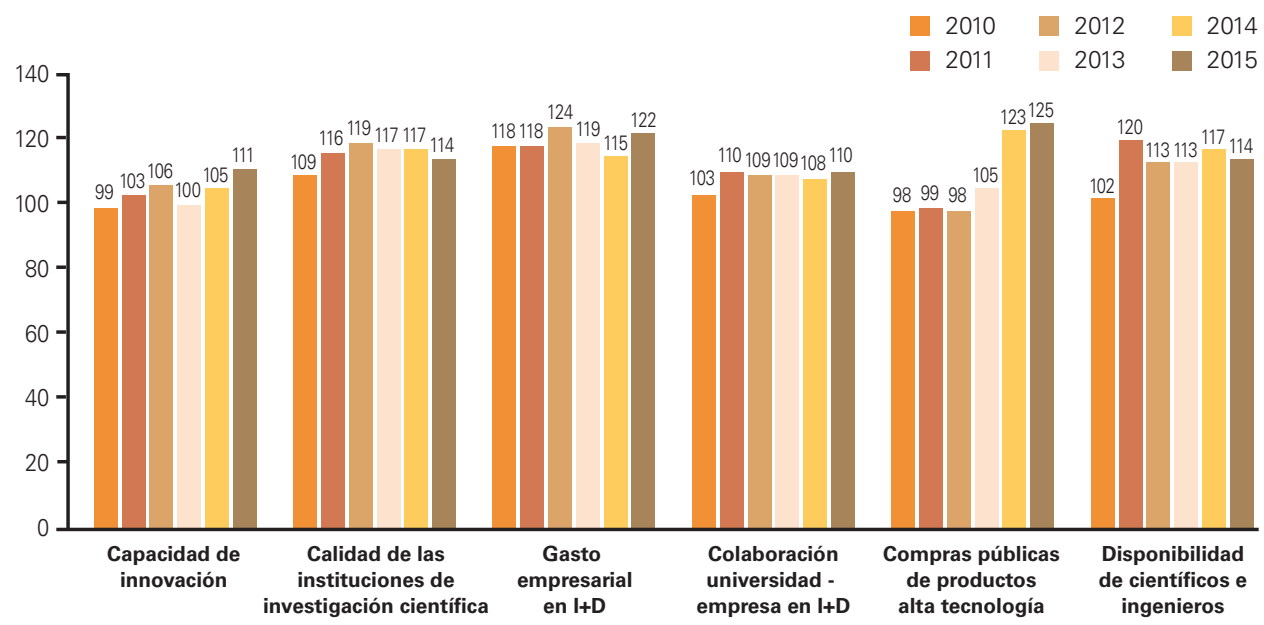

Fuente: elaboración propia a partir del World Economic Forum (2016).

De esta manera, y de forma global, Perú ha presentado una caída en el ranking mundial del pilar de innovación (ver gráfico 6), yendo del puesto 113 en 2010 al puesto 119 en 2015. A modo de ejemplo, solo tomando en cuenta a otros dos países para el análisis comparativo, tenemos que Kazajistán, en 2010, estaba en el puesto 116 del ranking mundial de innovación, por detrás de Perú y Chile. Sin embargo, para el año 2016 podemos apreciar que ocupa el puesto 59 de dicho ranking, superando a Chile (puesto 63) y Perú (puesto 119).

Por tanto, en estos últimos seis años hemos asistido a un retroceso en este pilar en comparación con otros países del ranking mundial de innovación.

\section{Gráfico 7: Pilar de innovación en países seleccionados (WEF, 2010-2015)}

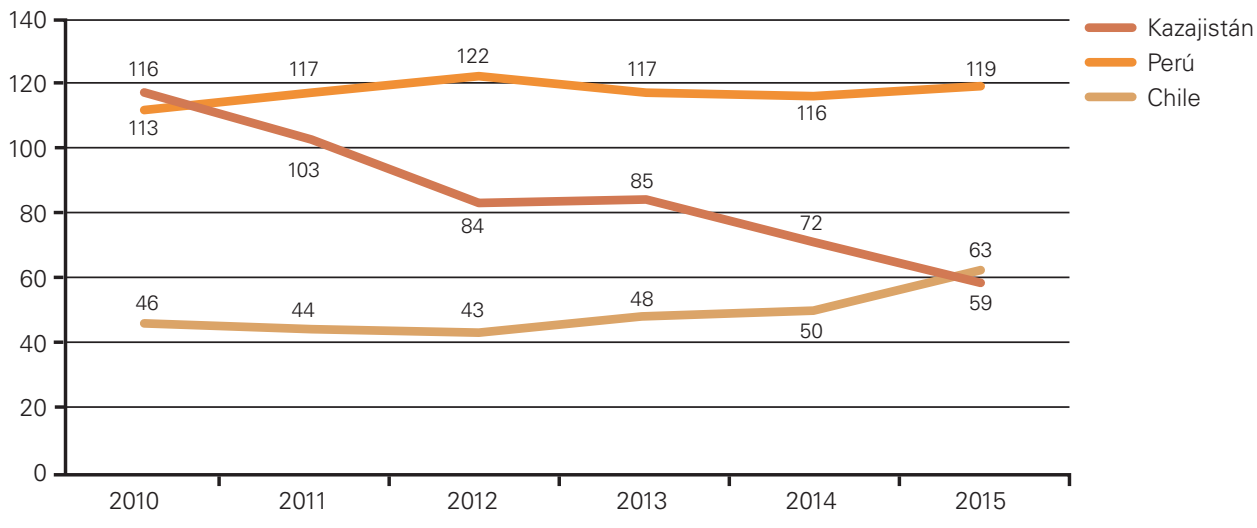

Fuente: elaboración propia a partir del World Economic Forum (2016).

En este punto, es importante mencionar que el número de países que conforman la muestra del $\mathrm{GCl}$ ha variado en el tiempo en función de la información disponible (por ejemplo, en 2010 la muestra estaba conformada por 142 países y en 2015 por 138 países). A pesar de ello, consideramos que esta variación no influye en los resultados obtenidos. 
Finalmente, a modo de resumen, podemos afirmar que los indicadores de innovación, tanto del Gll como del GCl, nos aportan una clara idea del estado del desarrollo de nuestro ecosistema de innovación en los últimos años. Sin embargo, consideramos que en dichos reportes se emplea una serie de indicadores sintéticos que presentan algunas limitaciones a la hora de explicar información a nivel más desagregado; por ejemplo, no queda claro si el gasto empresarial en I+D tiene mayor peso a partir de la I+D interna o externa, o si los vínculos para la innovación están centrados en la relación universidad-empresa, en la relación centros de investigación-empresa o simplemente en la relación empresa-empresa, entre otras cuestiones. Estas dificultades podrían limitar el análisis detallado de la situación real del estado de la innovación en el Perú; por ello, para complementar nuestro análisis, tomamos en cuenta a la ENIIM para intentar conocer más a detalle el estado de la innovación del Perú.

\section{Encuesta nacional de innovación en la industria manufacturera (2012 y 2015)}

Desde 2012 el Ministerio de la Producción y el Ministerio de Economía y Finanzas del Perú, con el financiamiento y la asistencia técnica del Banco Interamericano de Desarrollo, encargó al INEI realizar cada tres años la ENIIM con el propósito de obtener información básica de los procesos de innovación en nuestro país. Para la selección de la muestra se ha tomado en cuenta una distribución por estratos, según departamentos del Perú y la división CIIU. De esta forma, en 2012 la muestra comprendía 1220 empresas y en 2015 contenía 1452 empresas.

A pesar de que la muestra ha cambiado de un estudio a otro, podemos apreciar que, en general la proporción de empresas que realizan actividades de innovación ${ }^{2}$ se mantiene sin grandes cambios, aunque el número de empresas que realizan dichas actividades presenta una tendencia ligeramente creciente en 2015 con respecto a la encuesta de 2012 (ver gráfico 7). No obstante, hay algunas actividades de innovación que las empresas

2. En la ENIIM se considera como actividad de innovación a las siguientes: I+D interna (todo trabajo de creación realizado dentro de la empresa de forma sistemática con el objetivo de aumentar el volumen de conocimientos y desarrollar bienes, servicios o procesos nuevos o significativamente mejorados); adquisición de I+D externa ladquisición o financiación de las mismas actividades indicadas en la I+D interna, pero realizadas por un grupo de investigadores, institución o empresa de investigación con el acuerdo de que los resultados serán de propiedad total o parcial de la empresa contratante); adquisición de bienes de capital (incorporación de maquinarias, herramientas o edificios vinculados a la introducción de mejoras y/o nuevos bienes, servicios o procesos sin considerar reemplazo de equipamiento); adquisición de hardware (adquisición o alquiler de elementos de hardware específicamente destinados a introducir productos — bienes o servicios - o procesos nuevos o significativamente mejorados); adquisición de software (adquisición o alquiler de software específicamente destinado a introducir productos - bienes o servicios_o procesos nuevos o significativamente mejorados); transferencia de tecnología (transferencia de conocimiento sistemático para la elaboración de un producto — bien o servicio—, la aplicación de un proceso o la prestación de un servicio, contribuyendo al desarrollo de sus capacidades, adquisición de derechos de uso de patentes, licencias, marcas, diseño, know-how y también incluye asistencia técnica, consultorías y otros servicios contratados a terceros); diseño e ingeniería industrial (incluye todas las preparaciones técnicas para planificar y diseñar, implantar y controlar eficientemente organizaciones integradas por personas, materiales, equipos e información con la finalidad de asegurar el mejor desempeño de sistemas relacionados con la producción y administración de bienes y servicios); capacitación para actividades de innovación (capacitación interna o externa del personal de la empresa destinada a innovar -introducir nuevos y/o mejorados productos y procesos-. estudios de mercados para introducción de innovaciones - actividades vinculadas a la introducción en el mercado de bienes o servicios nuevos significativamente mejorados-). 
de manufacturas vienen realizando en menor medida, tales como la transferencia de tecnología, el diseño e ingeniería, la capacitación de personal y los estudios de mercado. En síntesis, las actividades de innovación que las empresas manufactureras menos realizan son la I+D interna, la transferencia de tecnología y los estudios de mercado, en ese orden de importancia.

\section{Gráfico 8: Empresas por actividades de innovación en la industria manufacturera, 2012-2015 (en porcentaje)}

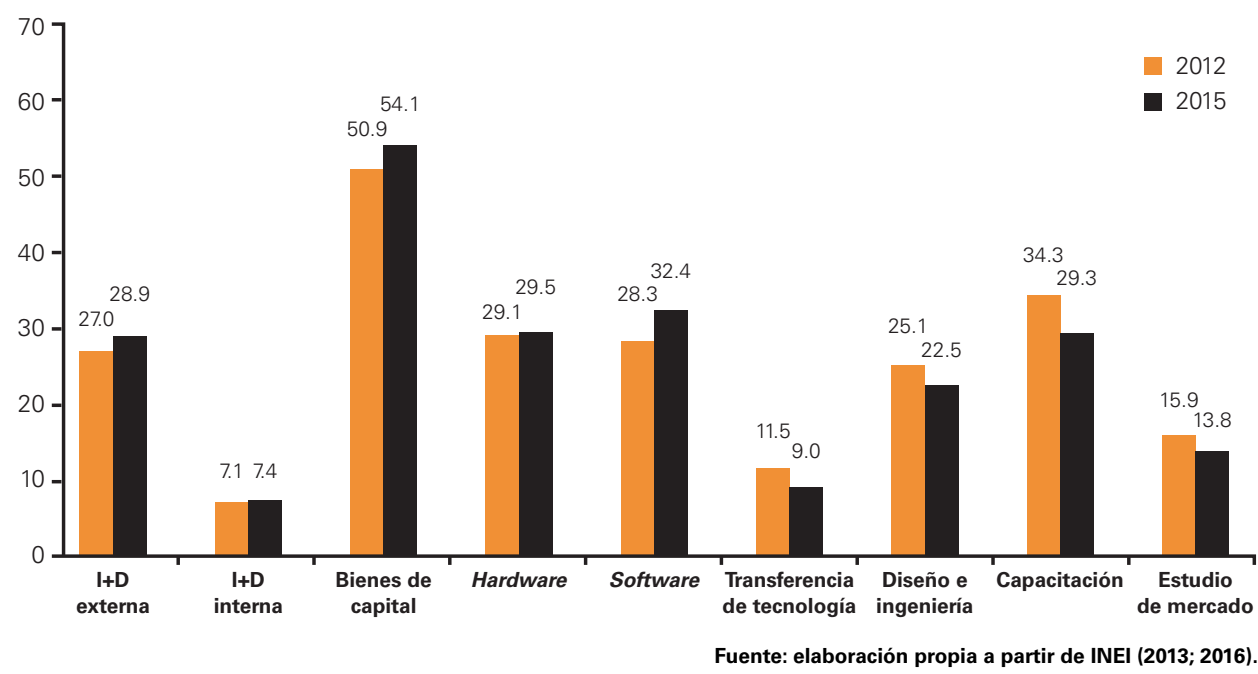

Por otro lado, si nos centramos en el monto invertido en las actividades de innovación, podemos apreciar que, si comparamos ambas encuestas, encontramos que la adquisición de bienes de capital ${ }^{3}$ es la actividad de innovación en la que más invierten las empresas de manufacturas (ver gráfico 8).

Asimismo, otras actividades de innovación por las que también los empresarios han aumentado su inversión son las actividades de I+D externa y la adquisición de software. Las demás actividades de innovación han experimentado una disminución en su inversión por parte de las empresas en 2015 con respecto a 2012.

3. Debemos ser cautelosos con la dependencia de la tecnología (conocimiento) foráneo, ya que el aumento de la dependencia externa puede tener un impacto negativo para la acumulación del conocimiento interno de las empresas (Itami, 2010; Seclén, 2015). 
(en porcentaje)

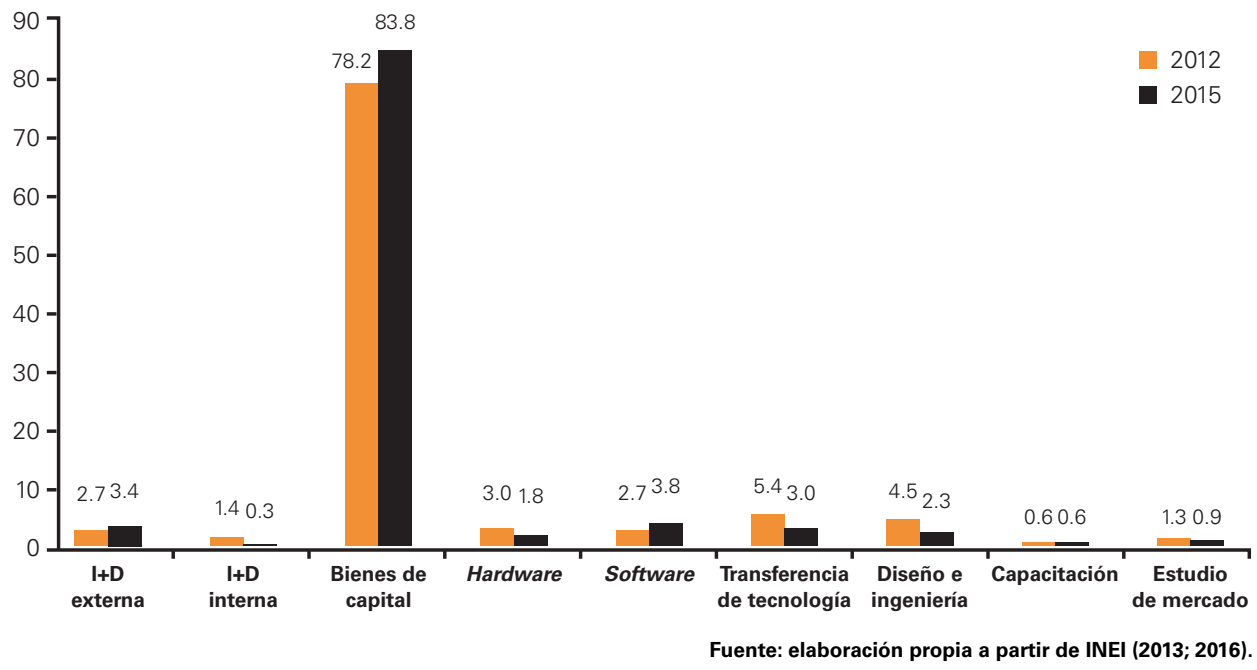

De otro lado, las actividades de innovación en las que las empresas manufactureras menos invierten son las actividades de I+D interna, la capacitación del personal y los estudios de mercado, en ese orden de importancia. Recordemos que todas estas actividades están ligadas a la capacidad de absorción del conocimiento y también a la colaboración para la innovación. En cualquier caso, podemos intuir a partir de lo observado que las relaciones más frecuentes están ligadas a la capacidad de producción, donde los proveedores de bienes de capital y de software adquieren gran relevancia.

Ahora bien, poniendo el foco de análisis en la encuesta nacional de innovación de 2015, encontramos que la inversión en actividades de innovación presenta una relación positiva con el tamaño empresarial (ver gráfico 9). Es decir, a medida que la empresa disminuye en su tamaño — de acuerdo al número de trabajadores—, también decrece la inversión en sus actividades de innovación en su conjunto.

Gráfico 10: Tamaño empresarial e inversión en actividades de innovación en la industria manufacturera, 2015 (Nuevos Soles)

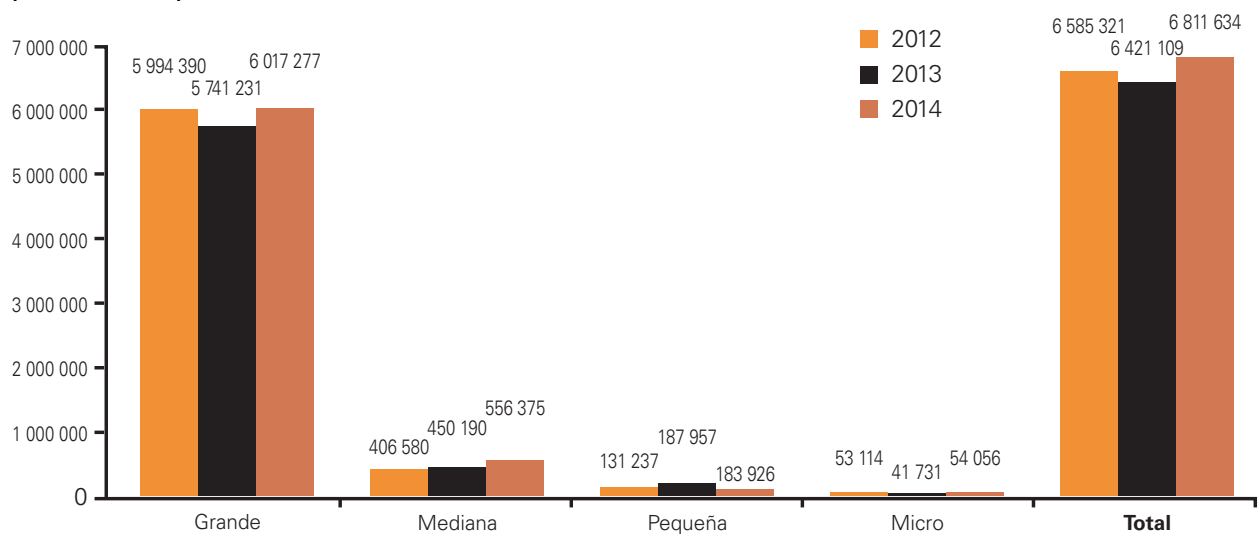


En cuanto a las actividades de innovación de las MIPYMES, en 2012, aparte de invertir en bienes de capital, encontramos que tanto las medianas como las pequeñas empresas orientan sus inversiones principalmente a actividades de I+D externa y actividades de diseño e ingeniería industrial (ver gráfico 10). Las microempresas, por su parte, orientaron sus inversiones principalmente en actividades de I+D externa y en adquisición de software.

\section{Gráfico 11: MIPYMES manufactureras e inversión en actividades de innovación (2012)}

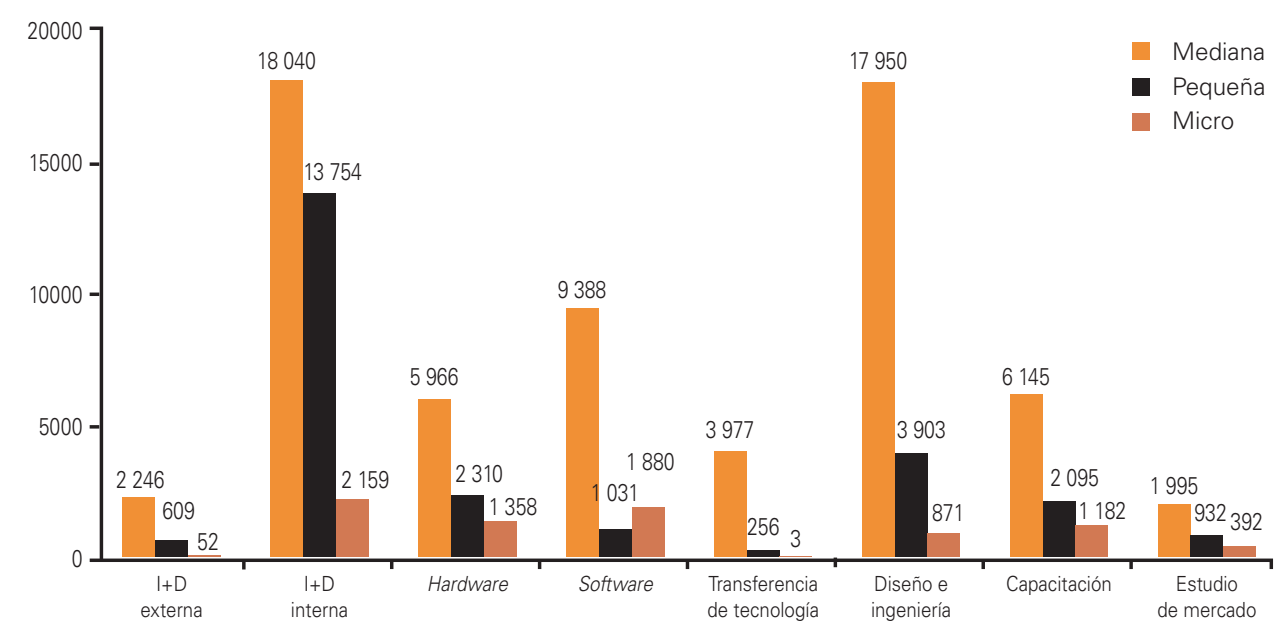

Fuente: elaboración propia a partir de INEI (2016).

En 2013, aparte de invertir en bienes de capital (ver gráfico 11), las empresas medianas y las microempresas orientaron sus inversiones principalmente a actividades de I+D externa y actividades de diseño e ingeniería industrial, mientras que las pequeñas empresas destinaron sus inversiones principalmente a actividades de I+D externa y de capacitación para actividades de innovación.

Gráfico 12: MIPYMES manufactureras e inversión en actividades de innovación (2013)

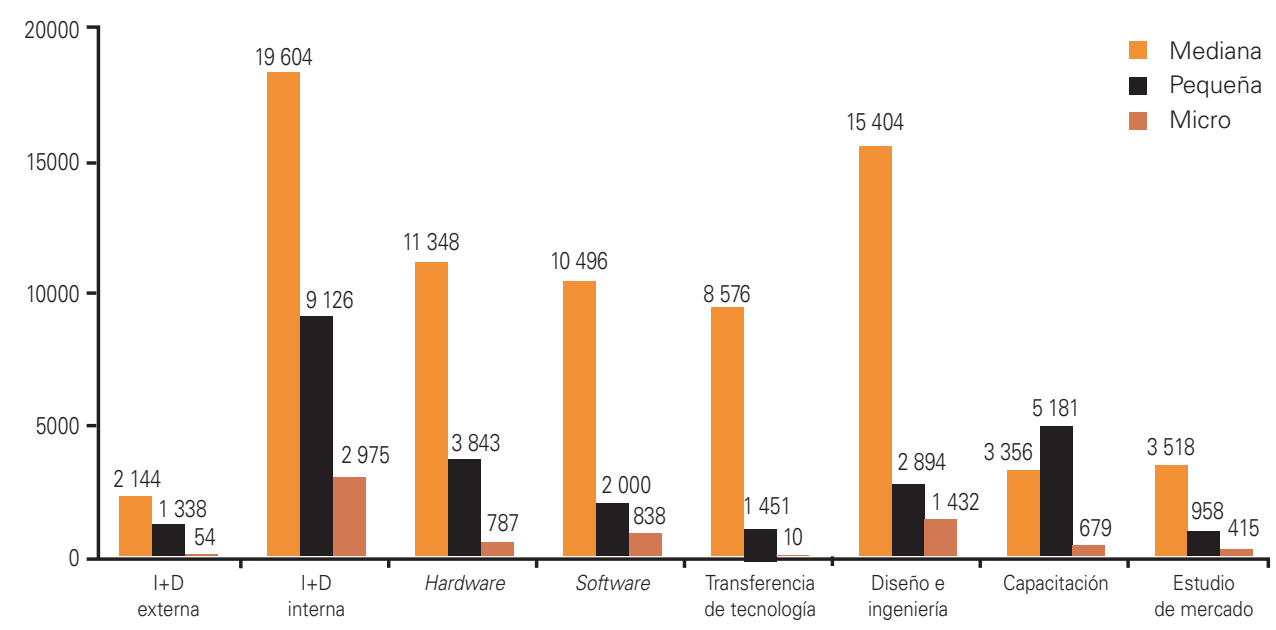

Fuente: elaboración propia a partir de INEI (2016). 
Finalmente, en 2014, aparte de invertir en bienes de capital, las medianas empresas orientaron sus inversiones principalmente a la adquisición de hardware. Las pequeñas empresas, por su parte, destinaron sus inversiones principalmente a la adquisición de software. Finalmente, las microempresas destinaron sus inversiones principalmente a actividades de I+D externa.

\section{Conclusiones}

El propósito de los indicadores sintéticos de innovación ha sido ofrecer una visión panorámica del estado de la innovación de los países para poder realizar un benchmarking de la situación a nivel global. Sin embargo, a nivel micro, parece ser que su aporte es más limitado.

A pesar de ello, ambos reportes hacen patente la falta de colaboración o vínculos para la innovación, lo cual nos hace intuir que la desconfianza y/o aversión al riesgo está presente en las empresas e instituciones peruanas, por lo que el ecosistema de innovación (en parte) se encuentra desarticulado (con ello perdemos fuelle) y otros países corren más rápido en la carrera hacia la competitividad y el crecimiento a partir de la innovación.

Del análisis de las encuestas de 2012 y 2015, encontramos que la actividad de innovación en la que las empresas de manufacturas más invierten es la adquisición de bienes de capital, lo cual está muy bien para ponerse al día (reducir costos unitarios de producción, mejorar y optimizar procesos, etcétera.); sin embargo, todo ello es temporal y no sostenible debido esencialmente al ritmo del avance de la ciencia y la tecnología.

Por otro lado, las actividades de innovación en las que las empresas manufactureras peruanas invierten menos son las actividades de I+D interna y de capacitación del personal, actividades ligadas a la construcción y/o potenciación de la capacidad de absorción del conocimiento y a la formación de vínculos para la innovación. De esta forma, la dependencia tecnológica es la estrategia más empleada por los empresarios peruanos, ya sea a partir de la adquisición de bienes de capital como de la adquisición de actividades de I+D externa, lo cual indica que las empresas (medianas, pequeñas y microempresas) no consideran relevante crear o acumular su propio conocimiento interno.

Quizás una explicación al respecto la podríamos encontrar en que carecen de incentivos para invertir en I+D+i interna debido principalmente a sus propias limitaciones competitivas, tales como: reducida dimensión, falta de capacidades para absorber el conocimiento, escasas relaciones o vínculos con otras organizaciones (públicas o privadas) para estos fines, falta de conocimiento sobre gestión de la innovación y el uso de herramientas para sistematizar y/o provocar las innovaciones, entre otras razones.

Finalmente, sería conveniente elaborar indicadores propios ajustados a nuestro contexto que se complementen con los indicadores que existen en la actualidad $y$, en particular, que midan la gestión de la innovación de las organizaciones. 


\section{bibliografía}

\section{Dutta, Soumitra \& Bruno}

2013 Lanvin (eds.)

The Global Innovation Index 2013: The Local Dynamics of Innovation. Ginebra, Ítaca y Fontainebleau: Cornell University, INSEAD \& WIPO. Disponible en: <http:// www.wipo.int/edocs/pubdocs/en/ economics/gii/gii 2013.pdf>

\section{Dutta, Soumitra; Bruno Lanvin} \& Sasha Wunsch-Vincent (eds.) The Global Innovation Index 2016: Winning with Global Innovation. Ginebra, Ítaca y Fontainebleau: Cornell University, INSEAD \& WIPO. Disponible en: <http://www.wipo.int/ edocs/pubdocs/en/wipo pub gii 2016.pdf>.

\section{OCDE}

OECD Reviews of Innovation Policy: Peru 2011. París: OECD Publishing.

\section{INEI}

Encuesta nacional de innovación en la industria manufacturera 2012. Lima: INEI.

\section{INEI}

Encuesta nacional de innovación en la industria manufacturera 2015. Lima: INEI.

\section{Ísmodes, Eduardo}

2016
2010

Dos dinámicas de conocimiento para la innovación. En BBVA (ed.), Innovación perspectivas para el siglo XXI (pp. 73-81). Madrid: BBVA. Disponible en: <https:// www.bbvaopenmind.com/articulo/ dos-dinamicas-de-conocimiento-para-lainnovacion/?fullscreen=true $>$.

\section{Schwab, Klaus}

The Global Competitiveness Report 20112013. Ginebra: World Economic Forum.

The Global Competitiveness Report 20112014. Ginebra: World Economic Forum

The Global Competitiveness Report 20112015. Ginebra: World Economic Forum

The Global Competitiveness Report 20112016. Ginebra: World Economic Forum

The Global Competitiveness Report 20112017. Ginebra: World Economic Forum.

\section{Seclén, Jean-Pierre} Retos para la industria peruana en el siglo XXI. Revista Enfoque, 1 (1), pp. 45-64. 